\title{
Towards predictive biomarkers for immunotherapy response in breast cancer patients
}

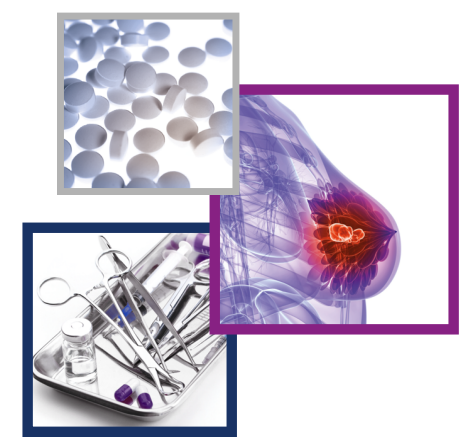

\author{
Leonie Voorwerk ${ }^{1}$, Marije Kat ${ }^{1}$ \& Marleen Kok*,1,2 \\ ${ }^{1}$ Department of Molecular Oncology \& Immunology, The Netherlands Cancer Institute Amsterdam, Amsterdam, The Netherlands \\ ${ }^{2}$ Department of Medical Oncology, The Netherlands Cancer Institute Amsterdam, Amsterdam, The Netherlands \\ *Author for correspondence: Tel.: +31 20512 1908; m.kok@nki.nl
}

\section{Practice points}

- Although response rates to anti-PD(L)1 are moderate in breast cancer, durable responses are seen.

- No accurate biomarkers for the prediction of response to immunotherapy in breast cancer are available yet.

- Response rates to anti-PD(L)1 appear higher in triple-negative breast cancer (TNBC) and PDL1-positive breast cancer.

- Early data show that TNBC patients with a tumor harboring relatively high levels of tumor infiltrating lymphocytes have a better outcome after anti-PD(L)1.

- As seen in melanoma patients, TNBC patients with high serum lactate dehydrogenase levels are less likely to respond to anti-PD(L)1 monotherapy.

- Exploiting research on immune biomarkers in other cancer types can guide the analyses of samples of breast cancer patients treated with anti-PD(L)1 in order to find predictive biomarkers.

- It needs to be determined whether potential biomarkers predicting response to anti-PD(L)1 have solely predictive value or capture information on prognosis as well.

Immunotherapy using anti-PD(L)1 has revolutionized treatment for various tumor types. Early data have shown durable responses in a small subgroup of breast cancer patients. So far, the response rates appear higher for breast tumors that are triple negative, PDL1-positive and/or harbor high levels of immune cells. Both comprehensive analyses of the breast tumor microenvironment and exploiting research on biomarkers in other cancer types, such as melanoma and lung cancer, may contribute to the discovery of accurate biomarkers to select breast cancer patients for immunotherapy. Here we summarize key features of the breast tumor microenvironment as well as putative predictive biomarkers established in other tumor types. Insights from both fields can guide future studies to enable personalized breast cancer immunotherapy.

First draft submitted: 28 September 2017; Accepted for publication: 30 January 2018; Published online: 09 April 2018

Keywords: atezolizumab • avelumab • biomarker $\bullet$ breast cancer $\bullet$ checkpoint inhibitor $\bullet$ CTLA-4 $\bullet$ durvalumab • immunotherapy • ipilimumab • nivolumab • PD-1 • PD-L1 • pembrolizumab

Immunotherapy has revolutionized cancer treatment for various tumor types. The major breakthroughs are seen with antagonistic monoclonal antibodies directed against CTLA-4, PD-1 and PD-L1. Objective response rates (ORRs) with anti-PD(L)1 treatment range from 40\% in stage IV melanoma patients [1] to 19-23\% in stage IV non-small-cell lung cancer (NSCLC) [2-4] and 25\% in renal cell carcinoma (RCC) patients [5]. Importantly, durable responses are seen in patients responding to therapy. Recently, impressive progression-free survival (PFS) benefits of anti-PDL1 were seen in stage III NSCLC patients after chemoradiotherapy as compared with placebo [6] and longer recurrence-free survival with adjuvant anti-PD1 in stage III/IV melanoma as compared with anti-CTLA-4 [7].

Historically, breast cancer was long considered a low-immunogenic malignancy. However, accumulating data regarding the value of breast tumor-associated immune cells and early data on efficacy of immune checkpoint inhibition such as anti-PD(L)1 in metastatic breast cancer have led to reconsideration of this viewpoint. Anti$\mathrm{PD}(\mathrm{L}) 1$ is currently under investigation in breast cancer patients and promising response rates have been presented. In order to treat the right patients with the right immunomodulatory therapy, biomarkers are needed to predict

Future Medicine 


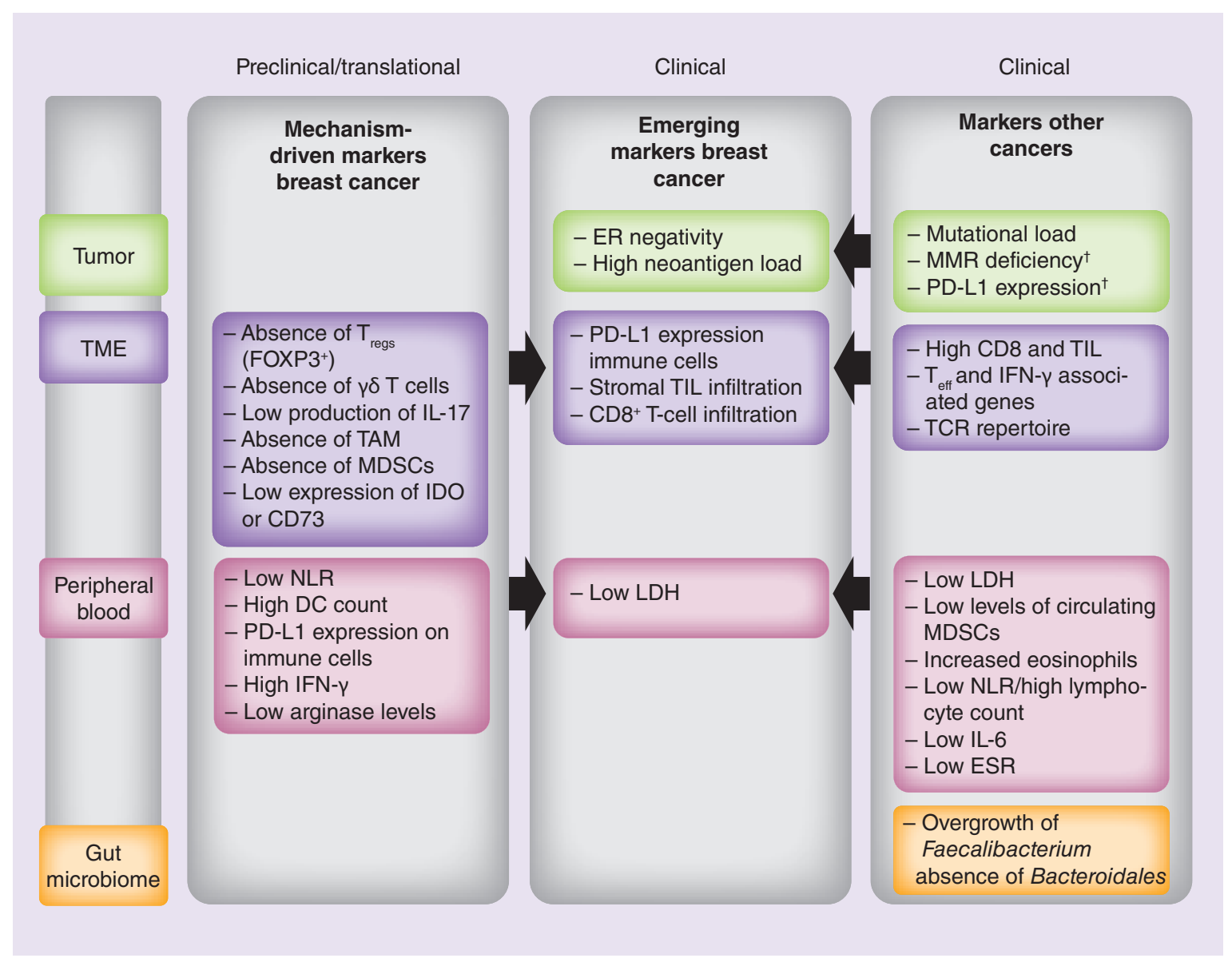

Figure 1. Opportunities for development of biomarkers to predict immunotherapy response in breast cancer. Currently, ongoing analyses of samples of breast cancer patients treated with anti-PD(L)1 have shown that ER-status, TIL, CD8 and LDH could be potential biomarkers (middle panel). New biomarker analyses can be guided by mechanism based preclinical or translational research (left panel) or from clinical trials with anti-PD(L)1 in other cancers, such as melanoma, NSCLC or bladder cancer (right panel). Information on these markers and references can be found in the text.

†US FDA approved biomarkers (PD-L1: $\geq 50 \%$ expression on tumor cells in NSCLC).

BC: Breast cancer; DC: Dendritic cell; ER: Estrogen receptor; ESR: Erythrocyte sedimentation rate; LDH: Lactate dehydrogenase; MDSC: Myeloid-derived suppressor cell; MMR: Mismatch repair; NLR: Neutrophil-to-lymphocyte ratio; NSCLC: Non-small-cell lung cancer; TAM: Tumor-associated macrophage; TCR: T-cell receptor; $T_{\text {eff }}$ : Effector T-cell; TIL: Tumor infiltrating lymphocyte; TME: Tumor microenvironment; $\mathrm{T}_{\text {regs }}$ : Regulatory T-cell.

response to immunotherapy. For breast cancer, no predictive biomarkers for immunotherapy are available yet. Indepth analyses of the breast cancer tumor microenvironment (TME) and the systemic immune response of breast cancer patients may help the identification of possible biomarkers. Furthermore, putative biomarkers found in other malignancies can guide the discovery of predictive biomarkers in breast cancer. In this review, we will describe the current status of biomarker research based on the small clinical trials evaluating anti-PD(L) 1 in metastatic breast cancer presented so far. In addition, we summarize previous preclinical and translational research on the anti-breast cancer immune response that might be crucial for response to anti-PD(L)1. Lastly, we will discuss putative predictive biomarkers established in other tumor types that might be important for the selection of breast cancer patients for immunotherapy. Figure 1 summarizes the opportunities for the development of biomarkers to predict immunotherapy response in breast cancer.

\section{First results of trials evaluating anti-PD(L)1 in breast cancer}

In 170 patients with triple-negative breast cancer (TNBC), pretreated with chemotherapy for metastatic disease and not preselected based on PD-L1 expression (KEYNOTE-086A), an ORR of 5\% was found upon treatment 
with anti-PD1 (pembrolizumab) [8]. In another cohort (KEYNOTE-012) of 27 patients with heavily pretreated PD-L1-positive metastatic TNBC (mTNBC), the ORR was 19\% [9]. In the KEYNOTE-86B cohort of 52 patients treated with anti-PD1 as first line of therapy for metastatic disease and with PD-L1-positive tumors, the ORR was 23\% [10]. In another Phase I study with 112 patients with mTNBC, treated with anti-PDL1 (atezolizumab), an ORR of $10 \%$ was seen [11]. In conclusion, in mTNBC response rates presented so far are around $5-23 \%$ and seem to be associated with PD-L1 expression and extend of previous chemotherapies.

On a smaller scale, anti-PD(L) 1 has been evaluated in metastatic ER-positive disease. Dirix et al. found an ORR of $3 \%$ in 72 patients after treatment with anti-PDL1 (avelumab) [12]. The KEYNOTE-028 study with 25 ER-positive metastatic breast cancer patients, all with PD-L1-positive tumors, revealed an ORR of 12\% [13]. Currently, many trials are ongoing in metastatic breast cancer evaluating the role of anti- $\mathrm{PD}(\mathrm{L}) 1$ in combination with chemotherapy. Anti-PDL1 plus nab-paclitaxel resulted in an ORR of $42 \%$ in $24 \mathrm{mTNBC}$ patients [14]. The combination of eribulin and anti-PD1 in 39 pretreated mTNBC patients induced responses in 33\% of the patients [15]. It still remains unclear whether this potential synergy between anti-PD $(\mathrm{L}) 1$ and chemotherapy is simply related to the direct effect on tumor growth or to proposed immunomodulatory aspects of chemotherapy [16]. Preliminary data presented at ESMO 2017 of the Phase II TONIC trial (NCT02499367) showed an ORR of $24 \%$ in 50 mTNBC patients after 2 weeks of pretreatment with either low-dose chemotherapy (cisplatin, doxorubicin and cyclophosphamide), radiotherapy or no pretreatment, followed by anti-PD1 (nivolumab) [17]. In-depth analyses of tumor biopsies are needed to unravel the potential immunomodulatory effect of chemotherapy/radiation on the breast TME.

Several neoadjuvant immunotherapy trials are ongoing in early breast cancer patients. In the TNBC cohort of the neoadjuvant adaptive I-SPY trial, Nanda et al. found three-times higher estimated complete response rates when combining anti-PD1 with paclitaxel, compared with paclitaxel monotherapy; 60 versus 20\%. Furthermore, the estimated complete response rate was significantly increased in ER-positive disease with the addition of anti-PD1 (34 vs 13\%) [18]. Schmid et al. described an ORR of $100 \%$ in ten early TNBC patients treated with carboplatin/nabpaclitaxel plus anti-PD1, followed by doxorubicin/cyclophosphamide (AC) and anti-PD1 [19]. Lastly, preliminary data in a third trial showed a pathological complete response rate of $71 \%$ in seven early TNBC patients treated with anti-PDL1 (durvalumab) plus nab-paclitaxel and AC [20].

\section{Potential biomarkers predicting outcome of anti-PD(L)1 in breast cancer}

As described above, so far, a limited number of patients were enrolled in clinical trials evaluating anti-PD(L) 1 in breast cancer. Moreover, these trials are not designed for the discovery and validation of biomarkers. In two studies PD-L1-positive TNBC had higher ORR as compared with patients with low expression of PD-L1 [11,12]. Furthermore, patients with at least 10\% stromal tumor infiltrating lymphocytes (sTIL) or 1.35\% CD8-positive cells (median used as cut-off) tended to have higher response rates [11]. Recently, Loi et al. confirmed the predictive value of sTIL in the KEYNOTE-086 cohorts, in which significantly higher levels of sTIL were seen in mTNBC patients responding to anti-PD1. This effect was even more pronounced in the KEYNOTE-86B cohort of PDL1-positive tumors that were not pretreated for metastatic disease. Of note, PD-L1 expression itself was not correlated with outcome after anti-PD1 [21].

Only a few studies have reported on potential biomarkers using peripheral blood. In the KEYNOTE-012 study, all five patients with a serum lactate dehydrogenase (LDH) level of above $800 \mathrm{U} / 1$ progressed within 8 weeks after study entry [9]. Also in the KEYNOTE-086 cohorts, LDH was associated with worse outcome after anti-PD1 treatment [21]. So far, no data are available on the predictive capacity of genetic characteristics in breast cancer, such as mutational load and gene expression. However, a slightly higher neoantigen load was seen in responders to combination therapy (anti-PDL1 plus anti-CTLA-4) in a small cohort of mTNBC and ER-positive patients [22].

\section{Breast tumor microenvironment}

Besides future unbiased analyses of side studies from the trials summarized above, insights gained in the last decades from preclinical as well as translational breast cancer research may be helpful on our way to personalized breast cancer immunotherapy. Here we discuss the most important components of the breast TME that could inform future analyses of samples of patients treated with anti-PD(L)1.

\section{Tumor intrinsic features}

Genomic instability is a hallmark of cancer and an important determinant of the immunogenicity of cancer cells. Accumulation of tumor-specific nonsynonymous mutations, called mutational load, can lead to the formation 
of peptides that are entirely absent from the normal human genome, so-called neoantigens. This can increase the 'foreignness' of the tumor and can thereby evoke an immune response [23]. In breast cancer, HER2-enriched tumors and TNBC exhibit a significantly higher mutation rate than ER-positive disease and are therefore more likely to harbor more neoantigens [24,25]. Copy number variants are caused by stable chromosomal rearrangements and occur early in breast cancer [26]. BRCA1/2-deficient breast cancers have impaired DNA double-strand break repair, which causes profuse copy number variant formation [27,28]. BRCA1-mutated cancers are associated with more lymphocytic infiltration as compared with a sporadic breast cancer control group [29]. Recently, Nolan et al. confirmed that TNBC tumors in the presence of a germline BRCA1 mutation have more sTIL and higher PD-L1 expression, as compared with wild-type tumors [30]. In addition, in DNA damage response-deficient tumors, more infiltration of $\mathrm{CD}^{+}$and $\mathrm{CD}^{+} \mathrm{T}$ cells was seen [31].

Recently, it has been shown that response rates to anti-PD1 are strikingly high in mismatch repair deficient (MMRd) tumors independent of the origin of the cancer cell [32]. Microsatellite instability, caused by MMRd, results in rapid accumulation of somatic mutations. MMRd is rare in breast cancer, but is present in about $1-2 \%$ of cases [33]. Profound response to anti-PD1 was seen in a breast cancer patient with a MMRd tumor [34]. Importantly, microsatellite instability might be associated with lower survival rates as seen in a small cohort of breast cancer patients [35].

\section{Tumor infiltrating lymphocytes}

Considerable heterogeneity exists between mammary tumors with respect to tumor infiltrating lymphocyte (TIL) density and immune cell composition [36]. Higher lymphocyte infiltration is correlated with ER negativity and better prognosis in general [37,38]. Moreover, high TIL levels have been associated with improved responses to neoadjuvant chemotherapy [39]. A distinction can be made between intratumoral and stromal lymphocytes. Stromal lymphocytes are considered a more reliable biomarker than intratumoral lymphocytes, probably due to the poor reproducibility of intratumoral TIL [40]. The lymphocyte population in breast cancers is largely comprised of $\mathrm{CD}^{+}$cytotoxic $\mathrm{T}$ cells and $\mathrm{CD}_{4}{ }^{+} \mathrm{T}$-helper cells, followed by regulatory $\mathrm{T}$ cells and different subpopulations of $\mathrm{B}$ cells $[41,42]$. High $\mathrm{CD} 8^{+}$T-cell infiltration is a positive prognostic factor generally associated with TNBC and HER2-enriched lesions [43]. Different subpopulations of $\mathrm{CD}^{+} \mathrm{T}$ cells are present in the breast cancer TME, which include T-helper (Th) subsets, regulatory $\mathrm{T}$ cells $\left(\mathrm{T}_{\text {regs }}\right), \gamma \delta \mathrm{T}$ cells and follicular $\mathrm{T}$ cells [44,45]. The presence of Th17 cells in breast cancer was associated with good prognosis, although functionally both pro- and antitumor functions have been reported [46]. $\mathrm{T}_{\text {regs }}$ are $\mathrm{CD} 4^{+} \mathrm{CD} 25^{+} \mathrm{FOXP}^{+} \mathrm{T}$ cells with a crucial role in protecting tissues from damage due to inflammation and preventing autoimmunity [47]. The presence of $T_{\text {regs }}$ is negatively correlated with disease outcome in breast cancer [48]. A small subset of T cells is represented by the $\gamma \delta \mathrm{T}$ cells, which have an alternative T-cell receptor (TCR) that is able to recognize antigens in MHC-unrestricted fashion [49]. Like $\mathrm{T}_{\text {regs }}$, these $\gamma \delta \mathrm{T}$ cells exhibit a regulatory function and were correlated to advanced stages and worse survival in breast cancer [50,51]. Conversely, follicular $\mathrm{T}$ cells have an antitumor role since they were associated with better prognosis in HER2positive breast cancer [45]. Tumor-reactive $\mathrm{B}$ cells can produce antibodies that recognize neoantigen epitopes, which potentiates tumor elimination by $T$ cells and innate immune cells [52]. Tumor-evoked regulatory $B$ cells $\left(\mathrm{B}_{\text {regs }}\right)$ were shown to secrete immunosuppressive cytokines, which inhibited proliferation of $\mathrm{CD} 4^{+} \mathrm{T}$ cells. In addition, PD-L1 expression on B cells was shown to inhibit antitumor T-cell responses [53].

\section{Tumor infiltrating myeloid cells}

Tumor-associated macrophages (TAMs) are known to facilitate metastasis [54,55]. In breast cancer, TAMs have been associated with ER negativity, higher histological grade and poor prognosis [56]. Neutrophils reduce T-cell proliferation and therefore suppress effector function. Alongside $\gamma \delta \mathrm{T}$ cells, neutrophils have a role in promoting breast cancer metastasis [57]. In a mechanistic mouse study, it was shown that inflamed mammary tumors secrete chemokine IL-1 $\beta$, which attracts $\gamma \delta \mathrm{T}$ cells and induces them to produce anti-inflammatory cytokine IL-17 and G-CSF. Neutrophils are recruited by these factors and secrete inducible NOS, which in turn suppresses antitumor $\mathrm{CD}^{+}$T-cell effector function and therefore facilitates metastasis [57]. Lastly, myeloid-derived suppressive cells (MDSCs) are immature myeloid cells that exhibit immunosuppressive capacity. MDSCs inhibit effector functions of T cells, natural killer (NK) cells and dendritic cells (DCs), while stimulating protumor functions of $\mathrm{T}_{\text {regs }}$, TAMs and Th2 cells through various mechanisms. Infiltrating MDSCs have been associated with unfavorable outcome in breast cancer [58]. Of note, new insights concerning tumor infiltrating myeloid cells have also generated confusion due to, for example, the lack of consensus on phenotypic markers to detect polarized macrophages or MDSCs [59]. 


\section{Other features of immunosuppression in breast cancer}

A balance between stimulatory and inhibitory signals regulates T-cell activity. One of the most important immune checkpoints in peripheral immunological tolerance is governed by PD-1. This co-inhibitory immune receptor represses the effector function of cytotoxic $\mathrm{T}$ cells to prevent autoimmunity [60]. PD1 expression is induced on T cells upon TCR activation [61,62]. Besides activated T cells, also activated B cells, NK cells and several other immune lineages were shown to express PD1 [63]. PD-L1, also known as B7-H1, is broadly expressed among different populations of immune cells and certain tissues, whereas PD-L2 is mainly limited to DCs. Cytokine IFN- $\gamma$, secreted by activated T cells, is shown to induce expression of both PD-L1 and PD-L2 [64,65]. Remarkably, in breast cancer PD-L1 expression was mainly observed on immune cells as opposed to tumor cells in other cancers [66].

IFN- $\gamma$ secreted in the TME induces expression of IDO [67]. IDO was linked to high TIL density in breast cancer [68]. Conversely, in a breast cancer murine model, high IDO expression was reported to have an immunosuppressive function on T cells, and might interfere with immunotherapy efficacy [69]. IDO inhibition is currently being evaluated in Phase I clinical trials in combination with immune checkpoint blockade [70]. Lastly, CD73 is upregulated by $\mathrm{T}_{\text {regs }}$ as another immune escape mechanism [71]. As a result of enzymatic activity of CD73, the $\mathrm{A} 2 \mathrm{a}$ receptor $(\mathrm{A} 2 \mathrm{aR})$ on $\mathrm{T}$ cells is activated, which in turn enhances PD1 expression [72,73]. CD73 expression has been associated with poor outcome in TNBC [74]. Both CD73 and A2aR antagonist therapies synergize with PD1 blockade in mouse models [73,75].

\section{Systemic immune response in breast cancer}

In contrast to chemotherapy or targeted therapy, immunotherapy is not directed against the cancer cells but can modulate the patient's immune system. Emerging data in the field of clinical melanoma research show that circulating immunosuppressive cells or factors are associated with poor outcome after immunotherapy. Here we summarize the current knowledge on features of this systemic immune response in breast cancer.

\section{Circulating immune cells}

It was shown that breast cancer patients have decreased peripheral lymphocyte levels, but differences were only statistically significant for $\mathrm{CD}^{+}$and $\mathrm{CD}^{+} \mathrm{T}$ cells [76]. Compared with healthy controls, higher peripheral blood $\mathrm{T}_{\text {reg }}$ counts were found in breast cancer patients [77,78]. Elevated neutrophil-to-lymphocyte ratio as a measure for systemic inflammation has recently been associated with lower survival rates [79]. Peripheral blood DC counts, myeloid-derived DCs in particular, were shown to be consistently reduced in breast cancer patients [80]. In advanced breast cancer patients, DC count was dramatically lower as compared with early breast cancer, where more immature DCs were observed [80,81]. Finally, PD-L1 mRNA expression in immune cells detected in peripheral blood might be associated with disease progression [82]. Interestingly, circulating metastatic breast cancer cells expressing PD-L1 were also detectable [83].

\section{Secreted immunological mediators}

In cancer patients, predominance of Th2-related cytokines was observed in peripheral blood, whereas Th1 cytokines were decreased [84]. Significantly reduced levels of IFN- $\gamma$ (Th1 associated) were measured in breast cancer patients, suggesting that IFN- $\gamma$ production by lymphocytes is partially or completely impaired [76]. Arginase serum levels were found to be elevated and increased during disease progression in breast cancer patients [85]. Treatment with VEGF antagonist bevacizumab inhibited the infiltration of suppressive immune cells (i.e., $\mathrm{T}_{\text {regs }}$, TAMs and MDSCs) in a breast cancer xenograft model [86]. As discussed above, serum LDH levels are associated with outcome after antiPD1 in TNBC, but LDH does also correlate with disease outcome in cancer. This suggests that LDH might not be a pure predictive factor for breast cancer immunotherapy response, but also captures prognostic information [87]. Acidification of the TME, as a result of conversion of pyruvate into lactate by $\mathrm{LDH}$, has detrimental consequences for infiltrating T cells, such as impaired cytokine production and lytic activity [88].

\section{Putative biomarkers for immunotherapy in other malignancies}

Research in melanoma, bladder cancer and NSCLC has already given important insights into features of the TME that predict response to anti- $\mathrm{PD}(\mathrm{L}) 1$. This information can be exploited with the aim to identify putative predictive biomarkers in breast cancer. According to the 'cancer immunogram' presented by Blank et al., seven parameter classes can be used to describe the interaction between cancer and the immune system [89]. In this section, possible biomarkers found in other malignancies than breast cancer, will be described according to these classes. 


\section{Tumor foreignness}

In patients with MMRd tumors treated with anti-PD1, high response rates are seen, independent of cancer type [32]. This led to the US FDA approval of MMRd as a biomarker for immunotherapy.

A relationship was shown between high nonsynonymous mutation burden and improved response to antiPD1 in NSCLC patients [90]. The significance of mutational load as a predictive marker was also confirmed in bladder cancer [91]. Frameshift indel mutations, small insertions or deletions, were associated with better response to checkpoint inhibition in melanoma [92]. Furthermore, poor outcome after anti-CTLA-4 treatment was seen in patients with melanoma with tumor aneuploidy and higher burden of copy number loss [93,94]. Clonal neoantigens but not the subclonal mutations were predictive of response to anti-PD1 in NSCLC [95].

\section{General immune status}

Increases in peripheral blood lymphocyte count during treatment has been correlated to improved anti-CTLA-4 response, whereas a high neutrophil-to-lymphocyte ratio was associated with inferior outcome in anti-PD1-treated NSCLC patients [96,97]. Higher rates of MDSCs were associated with lower response rates in anti-CTLA- 4 refractory melanoma patients treated with anti-PD1 [98] and lower overall survival (OS) in melanoma patients treated with anti-CTLA-4 [99]. In addition, it was seen that responders to anti-CTLA- 4 had a higher increase of eosinophils after one infusion as compared with non-responders. Also, monocyte and neutrophil count at baseline were significantly lower in responders. A decrease in monocytic MDSCs, a reduction in PD-L1 expression on MDSCs seemed predictive of response to anti-CTLA- 4 in the same cohort [100].

Immune cell infiltration

Increased TILs and $\mathrm{CD}^{+} \mathrm{T}$ cells in particular have been linked with therapeutic efficacy of $\mathrm{PD}(\mathrm{L}) 1$ checkpoint blockade in different cancer types, especially in melanoma [101,102]. An increase in TIL after treatment and higher baseline levels of FOXP3 and IDO were associated with better clinical outcome in melanoma patients treated with anti-CTLA-4 [103]. Higher frequencies of FOXP3 $3^{+} \mathrm{CD}^{+} \mathrm{T}$ cells were also associated with better survival in melanoma patients treated with anti-CTLA-4 [104]. CTLA4 is known to be expressed on FOXP3 ${ }^{+} \mathrm{T}_{\text {regs }}$ and therefore might be important for anti-CTLA-4 efficacy [105]. FOXP3 in relation to response to anti-PD(L)1 has not been well described. In RCC patients treated with anti-PDL1 a subtle higher ratio of effector T cells to regulatory $\mathrm{T}$ cells was seen in responders, as measured by gene expression [106].

\section{T-cell checkpoints}

PD-L1 expression on tumors has been linked with better responses to immunotherapy across different cancers. However, PD-L1 expression does not appear to be a requisite for immunotherapeutic responses and PDL1-positive tumors are not always responsive $[107,108]$. Interestingly in some tumor types, such as bladder cancer, PD-L1 expression on immune cells appears to be more predictive of anti-PD(L) 1 response than its expression on tumor cells $[108,109]$, as might be the case for breast cancer.

\section{Soluble inhibitors}

Low baseline levels of the erythrocyte sedimentation rate were associated with better OS in melanoma patients treated with anti-CTLA-4 [110]. In a small cohort of 40 melanoma patients, high IL-6 levels were associated with worse outcome after anti-CTLA-4 treatment [96].

\section{Inhibitory tumor mechanisms}

LDH has been shown to inhibit function and survival of T cells and NK cells [111]. High levels of serum LDH are correlated with worse response to anti-CTLA- 4 in melanoma patients [110]. Furthermore, higher response rates were seen in melanoma patients with normal LDH treated with anti-PD1 [112]. Whether LDH is mainly a prognostic factor or also predictive for immunotherapy response needs to be further established.

\section{Immune infiltrate activation}

T-effector- and IFN-y-associated genes were associated with better OS in NSCLC patients treated with antiPDL1 [113]. This was also shown in melanoma patients that responded to anti-CTLA-4 or anti-PD1, in which higher expression levels of genes were found related to $\mathrm{CD} 8^{+} \mathrm{T}$ cells, $\gamma \delta \mathrm{T}$ and NK cells, and lower levels of genes associated with MDSCs and $\mathrm{T}_{\text {regs }}$ [114]. Higher IFN-y associated gene expression levels were associated with better 
response to anti-PDL1 in urothelial cell carcinoma and anti-CTLA-4 in melanoma [108,115]. Deep sequencing of the T-cell receptor in peripheral blood samples of melanoma patients showed a correlation between TCR repertoire and better OS after immune checkpoint blockade [116]. An increased clinical benefit to anti-CTLA-4 was observed in patients with higher baseline TCR diversity measured from peripheral blood of melanoma patients [117]. In addition, it was found that melanoma patients who responded to neoadjuvant anti-PD1 and anti-CTLA-4, had more newly detectable TCR clones after 6 weeks of treatment [118].

\section{Gut microbiome}

Accumulating evidence indicates that an overgrowth or absence of specific gut bacteria might predict response to immunotherapy. In mice with melanoma, Bifidobacterium was needed for anti-PDL1 response. After oral administration of this gut microbiome bacterium, tumors were reduced in size to the same extent as with antiPDL1 alone [119]. In patients with metastatic melanoma treated with anti-PD1, response was associated with a 'favorable' gut microbiome. More diversity, high abundance of Faecalibacterium and low abundance of Bacteroidales was associated with response to anti-PD1 and longer PFS. Fecal microbiome transplant of germ-free mice with feces obtained from melanoma patients responding to anti-PD1, resulted in reduced tumor growth [120]. Another study showed that NSCLC and RCC patients treated with antibiotics during the course of anti-PD1 had shorter PFS and/or OS, as compared with patients not treated with antibiotics. This effect remained statistically significant in a multivariate analysis including other prognostic markers. An association between the abundance of Akkermansia muciniphila and a favorable clinical outcome was seen. Fecal microbiome transplant with $A$. muciniphila in sarcoma bearing mice restored response to anti-PD1 after treatment with antibiotics [121].

\section{Future perspective}

Knowledge on the breast TME, the systemic immune response in breast cancer patients and biomarkers found in other malignancies can guide the search for new biomarkers in breast cancer. In addition, understanding the mechanisms of response to immunotherapy in breast cancer will be crucial to increase benefit, including cure and quality of life of our breast cancer patients. To obtain this knowledge, comprehensive analysis of different types of data is essential. Therefore, large scale acquisition of cancer tissue, peripheral blood and other biospecimens is critical for the development of a robust classifier that can be used in the clinic to predict response and toxicity of immunotherapy in early and metastatic breast cancer patients.

Financial \& competing interests disclosure

M Kok has received an unrestricted research grant from Bristol-Meyers-Squibb paid to the institute and a translational fellowship of the Dutch Cancer Society (KWF) paid to the institute. The authors have no other relevant affiliations or financial involvement with any organization or entity with a financial interest in or financial conflict with the subject matter or materials discussed in the manuscript apart from those disclosed.

No writing assistance was utilized in the production of this manuscript.

Acknowledgements

We apologize to colleagues whose work was not cited in this review due to space constraints.

\section{Open access}

This work is licensed under the Creative Commons Attribution 4.0 License. To view a copy of this license, visit http://creativecommons.org/licenses/by/4.0/

\section{References}

Papers of special note have been highlighted as: $\bullet$ of interest; $\bullet \bullet$ of considerable interest

1. Robert C, Long GV, Brady B et al. Nivolumab in previously untreated melanoma without BRAF mutation. N. Engl. J. Med. 372(4), 320-330 (2015).

2. Garon EB, Rizvi NA, Hui R et al. Pembrolizumab for the treatment of non-small-cell lung cancer. N. Engl. J. Med. 372(21), 2018-2028 (2015).

3. Borghaei H, Paz-Ares L, Horn L et al. Nivolumab versus docetaxel in advanced nonsquamous non-small-cell lung cancer. N. Engl. J. Med. 373(17), 1627-1639 (2015).

4. Gettinger S, Rizvi NA, Chow LQ et al. Nivolumab monotherapy for first-line treatment of advanced non-small-cell lung cancer. J. Clin. Oncol. 34(25), 2980-2987 (2016). 
5. Motzer RJ, Escudier B, McDermott DF et al. Nivolumab versus everolimus in advanced renal-cell carcinoma. N. Engl. J. Med. 373(19), 1803-1813 (2015).

6. Antonia SJ, Villegas A, Daniel D et al. Durvalumab after chemoradiotherapy in stage III non-small-cell lung cancer. N. Engl. J. Med. 377(20), 1919-1929 (2017).

7. Weber J, Mandala M, Del Vecchio M et al. Adjuvant nivolumab versus ipilimumab in resected stage III or IV melanoma. N. Engl. J. Med. 377(19), 1824-1835 (2017).

8. Adams S, Schmid P, Rugo HS et al. Phase II study of pembrolizumab (pembro) monotherapy for previously treated metastatic triple-negative breast cancer (mTNBC): KEYNOTE-086 cohort A. J. Clin. Oncol. 35(Suppl. 15), 1008 (2017).

9. Nanda R, Chow LQ, Dees EC et al. Pembrolizumab in patients with advanced triple-negative breast cancer: Phase IB KEYNOTE-012 study. J. Clin. Oncol. 34(21), 2460-2467 (2016).

- The KEYNOTE-012 is the first published clinical trial investigating anti-PD1 in breast cancer, showing durable responses in a subset of patients.

10. Adams S, Loi S, Toppmeyer D et al. Phase II study of pembrolizumab as first-line therapy for PD-L1-positive metastatic triple-negative breast cancer (mTNBC): preliminary data from KEYNOTE-086 cohort B. J. Clin. Oncol. 35(Suppl. 15), 1088 (2017).

11. Schmid P, Cruz C, Braiteh FS et al. Abstract 2986: atezolizumab in metastatic TNBC (mTNBC): long-term clinical outcomes and biomarker analyses. Cancer Res. 77(Suppl. 13), 2986 (2017).

12. Dirix LY, Takacs I, Jerusalem G et al. Avelumab, an anti-PD-L1 antibody, in patients with locally advanced or metastatic breast cancer: a Phase IB JAVELIN solid tumor study. Breast Cancer Res. Treat. 167 (3), 671-686 (2017).

13. Rugo H, Delord J-P, Im S-A et al. Abstract S5-07: preliminary efficacy and safety of pembrolizumab (MK-3475) in patients with PD-L1-positive, estrogen receptor-positive (ER+)/HER2-negative advanced breast cancer enrolled in KEYNOTE-028. Cancer Res. 76(Suppl. 4), S5-07-S05-07 (2016).

14. Adams S, Diamond JR, Hamilton EP et al. Phase IB trial of atezolizumab in combination with nab-paclitaxel in patients with metastatic triple-negative breast cancer (mTNBC). J. Clin. Oncol. 34(Suppl. 15), 1009-1009 (2016).

15. Tolaney S, Savulsky C, Aktan G et al. Abstract P5-15-02: Phase IB/II study to evaluate eribulin mesylate in combination with pembrolizumab in patients with metastatic triple-negative breast cancer. Cancer Res. 77(Suppl. 4), P5-15-02-P15-15-02 (2017).

16. Kersten K, Salvagno C, De Visser KE. Exploiting the Immunomodulatory properties of chemotherapeutic drugs to improve the success of cancer immunotherapy. Front. Immunol. 6, 516 (2015).

17. Kok M, Horlings HM, van De Vijver K et al. LBA 14 - adaptive Phase II randomized noncomparative trial of nivolumab after induction treatment in triple negative breast cancer: TONIC-trial. Ann. Oncol. 28(Suppl. 5), V605-V649 (2017).

18. Nanda R, Liu MC, Yau C et al. Pembrolizumab plus standard neoadjuvant therapy for high-risk breast cancer (BC): results from I-SPY 2. J. Clin. Oncol. 35(Suppl. 15), 506-506 (2017).

19. Schmid P, Park YH, Muñoz-Couselo E et al. Pembrolizumab (pembro) + chemotherapy (chemo) as neoadjuvant treatment for triple negative breast cancer (TNBC): preliminary results from KEYNOTE-173. J. Clin. Oncol. 35(Suppl. 15), 556-556 (2017).

20. Pusztai L, Silber A, Hofstatter EW et al. Safety of MEDI4736 (anti-PD-L1 antibody) administered concomitant with weekly nab-paclitaxel and dose dense doxorubicin/cyclophosphamide (ddAC) as neoadjuvant chemotherapy for stage I-III triple negative breast cancer (TNBC): a Phase I/II trial. J. Clin. Oncol. 35(Suppl. 15), 572-572 (2017).

21. Loi S, Adams S, Schmid P et al. LBA13 - relationship between tumor infiltrating lymphocyte (TIL) levels and response to pembrolizumab (pembro) in metastatic triple-negative breast cancer (mTNBC): results from KEYNOTE-086. Ann. Oncol. 28(Suppl. 5), V605-V649 (2017).

22. Santa-Maria CA, Kato T, Park J-H et al. Durvalumab and tremelimumab in metastatic breast cancer (MBC): immunotherapy and immunopharmacogenomic dynamics. J. Clin. Oncol. 35(Suppl. 15), 3052-3052 (2017).

23. Schumacher TN, Schreiber RD. Neoantigens in cancer immunotherapy. Science 348(6230), 69-74 (2015).

- Schumacher et al. clearly describe the mechanisms and consequences of neoantigen formation in tumors and the cytotoxic T-cell response directed to these neoantigens.

24. Shah SP, Roth A, Goya R et al. The clonal and mutational evolution spectrum of primary triple-negative breast cancers. Nature 486(7403), 395-399 (2012).

25. Network TCGA. Comprehensive molecular portraits of human breast tumours. Nature 490(7418), 61-70 (2012).

26. Wang Y, Waters J, Leung ML et al. Clonal evolution in breast cancer revealed by single nucleus genome sequencing. Nature 512(7513), 155-160 (2014).

27. van Beers EH, van Welsem T, Wessels LF et al. Comparative genomic hybridization profiles in human BRCA1 and BRCA2 breast tumors highlight differential sets of genomic aberrations. Cancer Res. 65(3), 822-827 (2005).

28. Natrajan R, Weigelt B, Mackay A et al. An integrative genomic and transcriptomic analysis reveals molecular pathways and networks regulated by copy number aberrations in basal-like, HER2 and luminal cancers. Breast Cancer Res. Treat. 121(3), 575-589 (2010). 
29. Lakhani SR, Jacquemier J, Sloane JP et al. Multifactorial analysis of differences between sporadic breast cancers and cancers involving BRCA1 and BRCA2 mutations. J. Natl Cancer Inst. 90(15), 1138-1145 (1998).

30. Nolan E, Savas P, Policheni AN et al. Combined immune checkpoint blockade as a therapeutic strategy for BRCA1-mutated breast cancer. Sci. Transl. Med. 9(393), pii:eaal4922 (2017).

31. Parkes EE, Walker SM, Taggart LE et al. Activation of STING-dependent innate immune signaling by S-phase-specific DNA damage in breast cancer. J. Natl Cancer Inst. 109(1), pii:djw199 (2017).

32. Le DT, Durham JN, Smith KN et al. Mismatch repair deficiency predicts response of solid tumors to PD-1 blockade. Science 357(6349), 409-413 (2017).

-. Based on this clinical trial, the US FDA approved MMRd as a biomarker for anti-PD1 (pembrolizumab), which is the first biomarker not linked to a certain tumor type.

33. Davies H, Morganella S, Purdie CA et al. Whole-genome sequencing reveals breast cancers with mismatch repair deficiency. Cancer Res. 77(18), 4755-4762 (2017).

34. Kok M, Horlings HM, Snaebjornsson P et al. Profound immunotherapy response in mismatch repair-deficient breast cancer. JCO Precis. Oncol. doi:10.1200/PO.17.0005 (2017) (Epub ahead of print).

35. Paulson TG, Wright FA, Parker BA, Russack V, Wahl GM. Microsatellite instability correlates with reduced survival and poor disease prognosis in breast cancer. Cancer Res. 56(17), 4021-4026 (1996).

36. Stanton SE, Adams S, Disis ML. Variation in the incidence and magnitude of tumor-infiltrating lymphocytes in breast cancer subtypes: a systematic review. JAMA Oncol. 2(10), 1354-1360 (2016).

37. Adams S, Gray RJ, Demaria S et al. Prognostic value of tumor-infiltrating lymphocytes in triple-negative breast cancers from two Phase III randomized adjuvant breast cancer trials: ECOG 2197 and ECOG 1199. J. Clin. Oncol. 32(27), 2959-2966 (2014).

38. Salgado R, Denkert C, Campbell C et al. Tumor-infiltrating lymphocytes and associations with pathological complete response and event-free survival in HER2-positive early stage breast cancer treated with lapatinib and trastuzumab: a secondary analysis of the NeoALTTO Trial. JAMA Oncol. 1(4), 448-454 (2015).

39. Mao Y, Qu Q, Zhang Y, Liu J, Chen X, Shen K. The value of tumor infiltrating lymphocytes (TILs) for predicting response to neoadjuvant chemotherapy in breast cancer: a systematic review and meta-analysis. PLoS ONE 9(12), e115103 (2014).

40. Salgado R, Denkert C, Demaria $S$ et al. The evaluation of tumor-infiltrating lymphocytes (TILs) in breast cancer: recommendations by an International TILs Working Group 2014. Ann. Oncol. 26(2), 259-271 (2015).

- The tumor-infiltrating lymphocyte working group provides clear guidelines on the scoring of stromal tumor-infiltrating lymphocytes in several stages of breast cancer.

41. Ruffell B, Au A, Rugo HS, Esserman LJ, Hwang ES, Coussens LM. Leukocyte composition of human breast cancer. Proc. Natl Acad. Sci. USA 109(8), 2796-2801 (2012).

42. Burugu S, Asleh-Aburaya K, Nielsen TO. Immune infiltrates in the breast cancer microenvironment: detection, characterization and clinical implication. Breast Cancer 24(1), 3-15 (2017).

43. Ali HR, Provenzano E, Dawson SJ et al. Association between $\mathrm{CD}^{+}$T-cell infiltration and breast cancer survival in 12,439 patients. Ann. Oncol. 25(8), 1536-1543 (2014).

44. Coventry BJ, Weightman MJ, Bradley J, Skinner JM. Immune profiling in human breast cancer using high-sensitivity detection and analysis techniques. JRSM Open 6(9), 2054270415603909 (2015).

45. Gu-Trantien C, Loi S, Garaud S et al. CD4 ${ }^{+}$follicular helper T-cell infiltration predicts breast cancer survival. J. Clin. Invest. 123(7), 2873-2892 (2013).

46. Yang L, Qi Y, Hu J, Tang L, Zhao S, Shan B. Expression of Th17 cells in breast cancer tissue and its association with clinical parameters. Cell Biochem. Biophys. 62(1), 153-159 (2012).

47. Fontenot JD, Gavin MA, Rudensky AY. Foxp3 programs the development and function of $\mathrm{CD} 4{ }^{+} \mathrm{CD} 25^{+}$regulatory T cells. Nat. Immunol. 4(4), 330-336 (2003).

48. Demir L, Yigit S, Ellidokuz $\mathrm{H}$ et al. Predictive and prognostic factors in locally advanced breast cancer: effect of intratumoral FOXP3 ${ }^{+}$ Tregs. Clin. Exp. Metastasis 30(8), 1047-1062 (2013).

49. Shin S, El-Diwany R, Schaffert S et al. Antigen recognition determinants of gammadelta T-cell receptors. Science 308(5719), 252-255 (2005).

50. Ma C, Zhang Q, Ye J et al. Tumor-infiltrating gammadelta T lymphocytes predict clinical outcome in human breast cancer. J. Immunol. 189(10), 5029-5036 (2012).

51. Peng G, Wang HY, Peng W, Kiniwa Y, Seo KH, Wang R-F. Tumor-infiltrating $\gamma \delta \mathrm{T}$ cells suppress T- and dendritic-cell function via mechanisms controlled by a unique toll-like receptor signaling pathway. Immunity 27(2), 334-348 (2007).

52. Kotlan B, Simsa P, Teillaud JL et al. Novel ganglioside antigen identified by B cells in human medullary breast carcinomas: the proof of principle concerning the tumor-infiltrating B lymphocytes. J. Immunol. 175(4), 2278-2285 (2005). 
53. Zhang Y, Morgan R, Chen C et al. Mammary-tumor-educated B cells acquire LAP/TGF- $\beta$ and PD-L1 expression and suppress antitumor immune responses. Int. Immunol. 28(9), 423-433 (2016).

54. Wyckoff JB, Wang Y, Lin EY et al. Direct visualization of macrophage-assisted tumor cell intravasation in mammary tumors. Cancer Res. 67(6), 2649-2656 (2007).

55. Valković T, Dobrila F, Melato M, Sasso F, Rizzardi C, Jonjić N. Correlation between vascular endothelial growth factor, angiogenesis and tumor-associated macrophages in invasive ductal breast carcinoma. Virchows Arch. 440(6), 583-588 (2002).

56. Mahmoud SM, Lee AH, Paish EC, Macmillan RD, Ellis IO, Green AR. Tumour-infiltrating macrophages and clinical outcome in breast cancer. J. Clin. Pathol. 65(2), 159-163 (2012).

57. Coffelt SB, Kersten K, Doornebal CW et al. IL-17-producing $\gamma \delta \mathrm{T}$ cells and neutrophils conspire to promote breast cancer metastasis. Nature 522(7556), 345-348 (2015).

- Coffelt et al. describe the underlying mechanism of mammary tumor metastasis formation in vivo by studying the role of $\gamma \delta \mathrm{T}$ cells and neutrophils in systemic inflammation.

58. Markowitz J, Wesolowski R, Papenfuss T, Brooks TR, Carson WE. Myeloid-derived suppressor cells in breast cancer. Breast Cancer Res. Treat. 140(1), 13-21 (2013).

59. Elliott LA, Doherty GA, Sheahan K, Ryan EJ. Human tumor-infiltrating myeloid cells: phenotypic and functional diversity. Front. Immunol. 8, 86 (2017).

60. Crawford A, Wherry EJ. The diversity of costimulatory and inhibitory receptor pathways and the regulation of antiviral T-cell responses. Curr. Opin. Immunol. 21(2), 179-186 (2009).

61. Nishimura H, Agata Y, Kawasaki A et al. Developmentally regulated expression of the PD-1 protein on the surface of double-negative (CD4-CD8-) thymocytes. Int. Immunol. 8(5), 773-780 (1996).

62. Vibhakar R, Juan G, Traganos F, Darzynkiewicz Z, Finger LR. Activation-induced expression of human programmed death-1 gene in T-lymphocytes. Exp. Cell Res. 232(1), 25-28 (1997).

63. Keir ME, Butte MJ, Freeman GJ, Sharpe AH. PD-1 and its ligands in tolerance and immunity. Annu. Rev. Immunol. 26, 677-704 (2008).

64. Freeman GJ, Long AJ, Iwai Y et al. Engagement of the PD-1 immunoinhibitory receptor by a novel B7 family member leads to negative regulation of lymphocyte activation. J. Exp. Med. 192(7), 1027-1034 (2000).

65. Latchman Y, Wood CR, Chernova T et al. PD-L2 is a second ligand for PD-1 and inhibits T-cell activation. Nat. Immunol. 2(3), 261-268 (2001).

66. Cimino-Mathews A, Thompson E, Taube JM et al. PD-L1 (B7-H1) expression and the immune tumor microenvironment in primary and metastatic breast carcinomas. Hum. Pathol. 47(1), 52-63 (2016).

67. Spranger S, Spaapen RM, Zha Y et al. Upregulation of PD-L1, IDO and T(regs) in the melanoma tumor microenvironment is driven by CD8 $\left(^{+}\right)$T cells. Sci. Transl. Med. 5(200), 200ra116 (2013).

68. Denkert C, von Minckwitz G, Brase JC et al. Tumor-infiltrating lymphocytes and response to neoadjuvant chemotherapy with or without carboplatin in human epidermal growth factor receptor 2-positive and triple-negative primary breast cancers. J. Clin. Oncol. 33(9), 983-991 (2015).

69. Yu J, Wang Y, Yan F et al. Noncanonical NF-kappaB activation mediates STAT3-stimulated IDO upregulation in myeloid-derived suppressor cells in breast cancer. J. Immunol. 193(5), 2574-2586 (2014).

70. Spira AI, Hamid O, Bauer TM et al. Efficacy/safety of epacadostat plus pembrolizumab in triple-negative breast cancer and ovarian cancer: Phase I/II ECHO-202 study. J. Clin. Oncol. 35(Suppl. 15), 1103-1103 (2017).

71. Deaglio S, Dwyer KM, Gao W et al. Adenosine generation catalyzed by CD39 and CD73 expressed on regulatory T cells mediates immune suppression. J. Exp. Med. 204(6), 1257-1265 (2007)

72. Antonioli L, Yegutkin GG, Pacher P, Blandizzi C, Haskó G. Anti-CD73 in cancer immunotherapy: awakening new opportunities. Trends Cancer 2(2), 95-109 (2016).

73. Allard B, Pommey S, Smyth MJ, Stagg J. Targeting CD73 enhances the antitumor activity of anti-PD-1 and anti-CTLA-4 mAbs. Clin. Cancer Res. 19(20), 5626-5635 (2013).

74. Loi S, Pommey S, Haibe-Kains B et al. CD73 promotes anthracycline resistance and poor prognosis in triple negative breast cancer. Proc. Natl Acad. Sci. USA 110(27), 11091-11096 (2013).

75. Mittal D, Young A, Stannard K et al. Antimetastatic effects of blocking PD-1 and the adenosine A2A receptor. Cancer Res. 74(14), 3652-3658 (2014).

76. Caras I, Grigorescu A, Stavaru C et al. Evidence for immune defects in breast and lung cancer patients. Cancer Immunol. Immunother. 53(12), 1146-1152 (2004).

77. Liyanage UK, Moore TT, Joo HG et al. Prevalence of regulatory T cells is increased in peripheral blood and tumor microenvironment of patients with pancreas or breast adenocarcinoma. J. Immunol. 169(5), 2756-2761 (2002). 
78. Wolf AM, Wolf D, Steurer M, Gastl G, Gunsilius E, Grubeck-Loebenstein B. Increase of regulatory T cells in the peripheral blood of cancer patients. Clin. Cancer Res. 9(2), 606-612 (2003).

79. Ethier JL, Desautels D, Templeton A, Shah PS, Amir E. Prognostic role of neutrophil-to-lymphocyte ratio in breast cancer: a systematic review and meta-analysis. Breast Cancer Res. 19(1), 2 (2017).

80. Della Bella S, Gennaro M, Vaccari M et al. Altered maturation of peripheral blood dendritic cells in patients with breast cancer. $B r$. J. Cancer 89(8), 1463-1472 (2003).

81. Pinzon-Charry A, Ho CS, Maxwell T et al. Numerical and functional defects of blood dendritic cells in early- and late-stage breast cancer. Br. J. Cancer 97(9), 1251-1259 (2007).

82. Kawaguchi K, Suzuki E, Yamaguchi A, Yamamoto M, Morita S, Toi M. Altered expression of major immune regulatory molecules in peripheral blood immune cells associated with breast cancer. Breast Cancer 24(1), 111-120 (2017).

83. Mazel M, Jacot W, Pantel K et al. Frequent expression of PD-L1 on circulating breast cancer cells. Mol. Oncol. 9(9), 1773-1782 (2015).

84. Goto S, Sato M, Kaneko R, Itoh M, Sato S, Takeuchi S. Analysis of Th1 and Th2 cytokine production by peripheral blood mononuclear cells as a parameter of immunological dysfunction in advanced cancer patients. Cancer Immunol. Immunother. CII 48(8), 435-442 (1999).

85. Polat MF, Taysi S, Polat S, Böyük A, Bakan E. Elevated serum arginase activity levels in patients with breast cancer. Surg. Today 33(9), 655-661 (2003).

86. Roland CL, Lynn KD, Toombs JE, Dineen SP, Udugamasooriya DG, Brekken RA. Cytokine levels correlate with immune cell infiltration after anti-VEGF therapy in preclinical mouse models of breast cancer. PLoS ONE 4(11), e7669 (2009).

87. Zhang J, Yao YH, Li BG, Yang Q, Zhang PY, Wang HT. Prognostic value of pretreatment serum lactate dehydrogenase level in patients with solid tumors: a systematic review and meta-analysis. Sci. Rep. 5, 9800 (2015).

88. Fischer K, Hoffmann P, Voelkl S et al. Inhibitory effect of tumor cell-derived lactic acid on human T cells. Blood 109(9), 3812-3819 (2007).

89. Blank CU, Haanen JB, Ribas A, Schumacher TN. Cancer immunology. The 'cancer immunogram'. Science 352(6286), 658-660 (2016).

-• This paper provides an informative framework for possible biomarkers and resistance mechanisms of immunotherapy.

90. Rizvi NA, Hellmann MD, Snyder A et al. Cancer immunology. Mutational landscape determines sensitivity to PD-1 blockade in non-small-cell lung cancer. Science 348(6230), 124-128 (2015).

91. Rosenberg JE, Hoffman-Censits J, Powles T et al. Atezolizumab in patients with locally advanced and metastatic urothelial carcinoma who have progressed following treatment with platinum-based chemotherapy: a single-arm, multicenter, Phase II trial. Lancet 387(10031), 1909-1920 (2016).

92. Turajlic S, Litchfield K, Xu H et al. Insertion-and-deletion-derived tumour-specific neoantigens and the immunogenic phenotype: a pan-cancer analysis. Lancet Oncol. 18(8), 1009-1021 (2017).

93. Davoli T, Uno H, Wooten EC, Elledge SJ. Tumor aneuploidy correlates with markers of immune evasion and with reduced response to immunotherapy. Science 355(6322), pii:eaaf8399 (2017).

94. Roh W, Chen PL, Reuben A et al. Integrated molecular analysis of tumor biopsies on sequential CTLA-4 and PD-1 blockade reveals markers of response and resistance. Sci. Transl. Med. 9(379), pii:eaah3560 (2017).

95. Mcgranahan N, Furness AJS, Rosenthal R et al. Clonal neoantigens elicit T-cell immunoreactivity and sensitivity to immune checkpoint blockade. Science 351(6280), 1463-1469 (2016).

96. Bjoern J, Juul Nitschke N, Zeeberg Iversen T, Schmidt H, Fode K, Svane IM. Immunological correlates of treatment and response in stage IV malignant melanoma patients treated with ipilimumab. Oncoimmunology 5(4), e1100788 (2016).

97. Bagley SJ, Kothari S, Aggarwal C et al. Pretreatment neutrophil-to-lymphocyte ratio as a marker of outcomes in nivolumab-treated patients with advanced non-small-cell lung cancer. Lung Cancer 106, 1-7 (2017).

98. Weber J, Gibney G, Kudchadkar R et al. Phase I/II study of metastatic melanoma patients treated with nivolumab who had progressed after ipilimumab. Cancer Immunol. Res. 4(4), 345-353 (2016).

99. Kitano S, Postow MA, Ziegler CG et al. Computational algorithm-driven evaluation of monocytic myeloid-derived suppressor cell frequency for prediction of clinical outcomes. Cancer Immunol. Res. 2(8), 812-821 (2014).

100. Gebhardt C, Sevko A, Jiang H et al. Myeloid cells and related chronic inflammatory factors as novel predictive markers in melanoma treatment with ipilimumab. Clin. Cancer Res. 21(24), 5453-5459 (2015).

101. Hamid O, Robert C, Daud A et al. Safety and tumor responses with lambrolizumab (anti-PD-1) in melanoma. N. Engl. J. Med. 369(2), 134-144 (2013).

102. Tumeh PC, Harview CL, Yearley JH et al. PD-1 blockade induces responses by inhibiting adaptive immune resistance. Nature 515(7528), 568-571 (2014).

103. Hamid O, Schmidt H, Nissan A et al. A prospective Phase II trial exploring the association between tumor microenvironment biomarkers and clinical activity of ipilimumab in advanced melanoma. J. Transl. Med. 9, 204 (2011). 
104. Martens A, Wistuba-Hamprecht K, Geukes Foppen M et al. Baseline peripheral blood biomarkers associated with clinical outcome of advanced melanoma patients treated with ipilimumab. Clin. Cancer Res. 22(12), 2908-2918 (2016).

105. Tang AL, Teijaro JR, Njau MN et al. CTLA4 expression is an indicator and regulator of steady-state $\mathrm{CD}^{+}{ }^{+}$FoxP3 ${ }^{+} \mathrm{T}_{\text {-cell homeostasis. }}$ J. Immunol. 181(3), 1806-1813 (2008).

106. Mcdermott DF, Sosman JA, Sznol M et al. Atezolizumab, an anti-programmed death-ligand 1 antibody, in metastatic renal cell carcinoma: long-term safety, clinical activity and immune correlates from a Phase IA study. J. Clin. Oncol. 34(8), 833-842 (2016).

107. Patel SP, Kurzrock R. PD-L1 expression as a predictive biomarker in cancer immunotherapy. Mol. Cancer Ther. 14(4), 847-856 (2015).

108. Herbst RS, Soria JC, Kowanetz M et al. Predictive correlates of response to the anti-PD-L1 antibody MPDL3280A in cancer patients. Nature 515(7528), 563-567 (2014).

109. Powles T, Eder JP, Fine GD et al. MPDL3280A (anti-PD-L1) treatment leads to clinical activity in metastatic bladder cancer. Nature 515(7528), 558-562 (2014).

110. Kelderman S, Heemskerk B, Van Tinteren H et al. Lactate dehydrogenase as a selection criterion for ipilimumab treatment in metastatic melanoma. Cancer Immunol. Immunother. 63(5), 449-458 (2014).

111. Brand A, Singer K, Koehl GE et al. LDHA-associated lactic acid production blunts tumor immunosurveillance by T and NK cells. Cell Metab. 24(5), 657-671 (2016).

112. Ribas A, Hamid O, Daud A et al. Association of pembrolizumab with tumor response and survival among patients with advanced melanoma. JAMA 315(15), 1600-1609 (2016).

113. Fehrenbacher L, Spira A, Ballinger M et al. Atezolizumab versus docetaxel for patients with previously treated non-small-cell lung cancer (POPLAR): a multicenter, open-label, Phase II randomized controlled trial. Lancet 387(10030), 1837-1846 (2016).

114. Charoentong P, Finotello F, Angelova $\mathrm{M}$ et al. Pan-cancer immunogenomic analyses reveal genotype-immunophenotype relationships and predictors of response to checkpoint blockade. Cell Rep. 18(1), 248-262 (2017).

115. Ji RR, Chasalow SD, Wang L et al. An immune-active tumor microenvironment favors clinical response to ipilimumab. Cancer Immunol. Immunother. 61(7), 1019-1031 (2012).

116. Cha E, Klinger M, Hou Y et al. Improved survival with T-cell clonotype stability after anti-CTLA-4 treatment in cancer patients. Sci. Transl. Med. 6(238), 238ra270 (2014).

117. Postow MA, Manuel M, Wong P et al. Peripheral T-cell receptor diversity is associated with clinical outcomes following ipilimumab treatment in metastatic melanoma. J. Immunother. Cancer 3, 23 (2015).

118. Rozeman EA, Blank CU, Akkooi ACJV et al. Neoadjuvant ipilimumab + nivolumab (IPI + NIVO) in palpable stage III melanoma: updated data from the OpACIN trial and first immunological analyses. J. Clin. Oncol. 35(Suppl. 15), 9586 (2017).

119. Sivan A, Corrales L, Hubert N et al. Commensal Bifidobacterium promotes antitumor immunity and facilitates anti-PD-L1 efficacy. Science 350(6264), 1084-1089 (2015).

120. Gopalakrishnan V, Spencer CN, Nezi L et al. Gut microbiome modulates response to anti-PD-1 immunotherapy in melanoma patients. Science 359(6371), 97-103 (2017).

121. Routy B, Le Chatelier E, Derosa L et al. Gut microbiome influences efficacy of PD-1-based immunotherapy against epithelial tumors. Science 359(6371), 91-97 (2017). 